\title{
Enhancement of fiber-SPR sensor utilizing graphene oxide
}

\begin{abstract}
A graphene oxide layer deposited on top of a thin gold film of a fiber optic surface plasmon resonance sensor is investigated. Comparison is made on the sensing performance of modified sensor and a standard gold coated fiber optic surface plasmon resonance sensor. The graphene oxide coated sensor shows high sensitivity of approximately $400 \mathrm{~nm} / \mathrm{RIU}$ to surrounding refractive index when tested with different liquids.
\end{abstract}

Keyword: Surface plasmon resonance; Graphene oxide; Miniature sensors; Fiber optic sensors 